\title{
MAGNETIC BRAKE CYLINDER TO ENHANCE TRAVERSE ABILITY AND ITS APPLICATION TO RESCUE ROBOTS
}

\author{
Eyri WATARI*, Hideyuki TSUKAGOSHI*, Yasuhiro KITAGAWA* and Ato KITAGAWA** \\ * Department of Mechanical and Control Engineering \\ Tokyo Institute of Technology \\ 2-12-1-S5-16 O-okayama, Meguro-ku, Tokyo, 152-8552 Japan \\ (E-mail: eyri.watari@cm.ctrl.titech.ac.jp)
}

\begin{abstract}
In this paper, a novel pneumatic cylinder is proposed, named Magnetic Brake Cylinder. It is composed of a tank, a pneumatic cylinder, and a permanent magnet, and with this, the piston is held by the attractive force of the magnet until the pressure inside the tank is very high, offering a higher driving power than an ordinary cylinder when using the same pressure source. In addition, a control method of its driving power is introduced. The developed actuator is then mounted into two types of rescue inspectors: a rolling and jumping rescue robot, which uses a cylinder to jump over obstacles, and a throw and collect rescue inspector, which deploys with the cylinder a child machine over high obstacles. Both developed prototypes showed higher jumping and throwing height than when using an ordinary cylinder, proving to be an option of effective enhancement of traversing ability for pneumatically powered robots.
\end{abstract}

\section{KEY WORDS}

Pneumatic cylinder, Rescue inspector

\section{INTRODUCTION}

In order to enhance the efficiency of searching survivors inside collapsed buildings after an earthquake, several types of rescue robots have being researched among these days. The demanding functions of a rescue robot are mainly three: ability of locomotion through rough terrains by remote control, sensing functions, and human-machine interface. Using existing technologies of sensing and transmitting systems, and having a useful rescue capacity, the enhancement of the obstacle traversing ability and the accessibility to the site of the robot is an important factor.

As for these rescue robots, it is possible to classify them into two major groups. One group is composed of robots which move their center of gravity continuously, and the other is composed of those which move their center of gravity in scattered pattern. The first group is represented mainly by legged robots, wheeled robots, snake type robots, and the hybrid robots composed of these types. Since their center of gravity moves continuously, it is simpler to increase the weight of the robot without losing much of the efficiency of traversing obstacles, and therefore, it is possible to mount several types of sensors. The second group is composed of robots that kick the ground performing a jump, or robots that deploys part of it or another robot over obstacles. These have not been applied to rescue in large scale as the first group is, but this group holds the possibility of showing high performance and agility which cannot be obtained by continuous locomotion of the center of gravity.

Therefore, this research concentrates on a pneumatic cylinder as the main actuator to realize the locomotion 
method based on moving the center of gravity in a scattered pattern. Then, at the same time that it shows limitation of traversing ability when composing with an ordinary cylinder, a new cylinder is proposed to overcome this limit.

In this paper, after showing the target locomotion methods, the limitation of the pneumatic cylinder is presented. Then, the Magnetic Brake Cylinder (MB Cylinder hereafter) is introduced. Its control method is also proposed. Finally, the application of the MB Cylinder to the target locomotion methods is showed.

\section{TARGET LOCOMOTION METHODS}

When inspecting inside buildings after disasters, the key issue is the locomotion function of the robot, since it must have ability to traverse obstacles such as furniture and debris. The authors have proposed locomotion methods based on the realization of the traverse action in short interval of time, the possibility of traversing obstacles regardless the shape, and enhancement of traversing height regardless the body size being small.

\section{Roll and Jump Mobile Inspector}

One simple locomotion method proposed by the authors is the Roll and Jump Mobile Inspector.[1] It consists of throwing the inspector inside the building, and this inspector uses wheels to efficiently roll on surfaces, it can jump over obstacles larger than its body, and it can also recover its posture from any landing position (Figure 1).

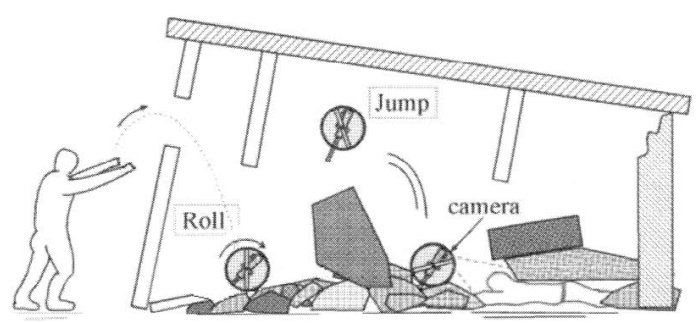

Figure 1 Concept of Roll and Jump Inspector

The rolling motion is driven by an electric motor, while the jump is driven by a pneumatic cylinder (Figure 2), and with this coupled drive the robot is able to traverse obstacles higher than three times its body size. In order to achieve a jumping height of $1 \mathrm{~m}$, due to limits of maximum air pressure and flow rate of air, the pneumatic cylinder is analyzed, providing an optimization method of its size.

The developed Roll and Jump Mobile Inspector, named Leg-in-Rotor-V, has $300 \mathrm{~mm}$ of diameter and $500 \mathrm{~mm}$ of length, and weights less than $2 \mathrm{~kg}$. it is equipped with a wireless microcamera and it is controlled remotely. Figure 3 shows the inspector traversing an obstacle of $1 \mathrm{~m}$ height.

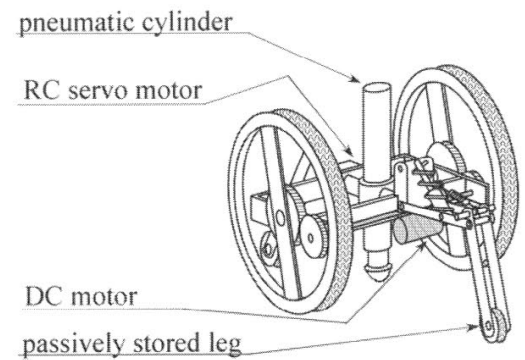

Figure 2 Basic structure of Jump and Roll Inspector

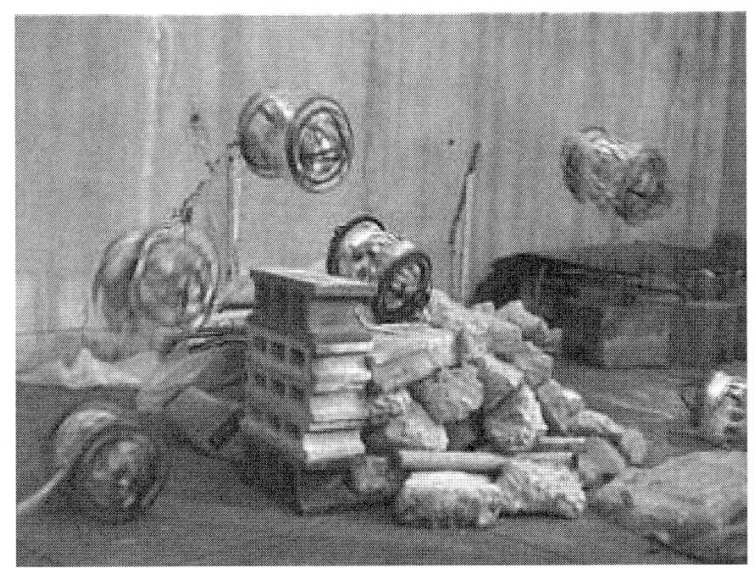

Figure 3 Performance of Leg-in-Rotor-V

However, if the ground conditions are too rough, or if it is soft as a mattress, the robot may not perform the jump as expected. For such situation, the following locomotion method is proposed.

\section{Throw \& Collect Type Rescue Inspector}

This method consists of the following sequence (Figure 4): after inserting the parent robot (or a pole with the mechanisms) into the site, this throws out a child machine, equipped with camera, and the inspection is performed while this child machine is been drawn back.[2] Using this method, the ground conditions do not affect the throw, which can be done many times into different directions, covering a wide search area.

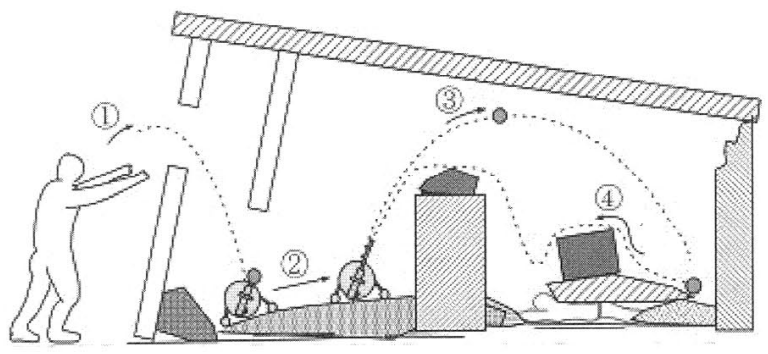

Figure 4 Concept of Throw \& Collect Inspector

The basic structure of the Throw \& Collect Type Rescue Inspector is shown in Figure 5. The parent robot 
(or pole) is equipped with a throwing device and a drawing device. In this case, a pneumatic cylinder is used for the throwing device.

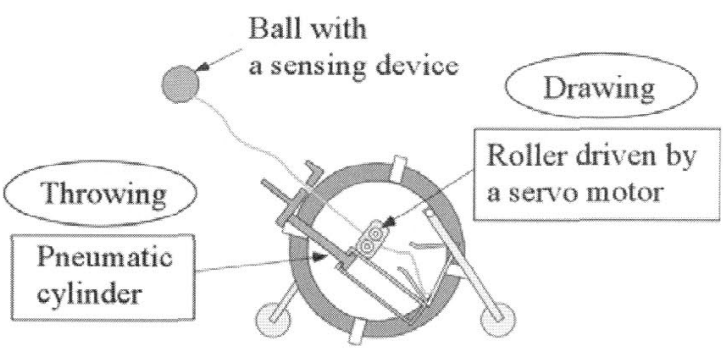

Figure 5 Structure of a Throw \& Collect Inspector

Figure 6 shows the drawing device, which consists of a pinch-roller mechanism (Figure 7). The wire which connects the child machine is pinched and pushed by the rollers into a taper case, and this wire is automatically stored in a spiral motion into the case. When performing a throw, the pinch-roller mechanism is opened, and the wire becomes free to be pulled out of the case.

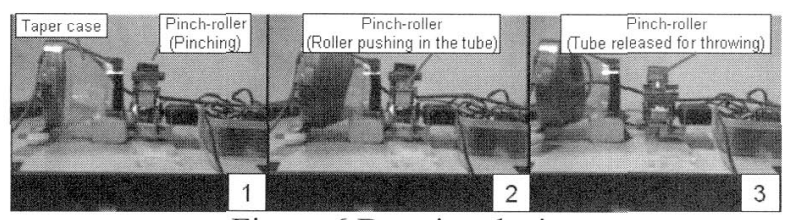

Figure 6 Drawing device

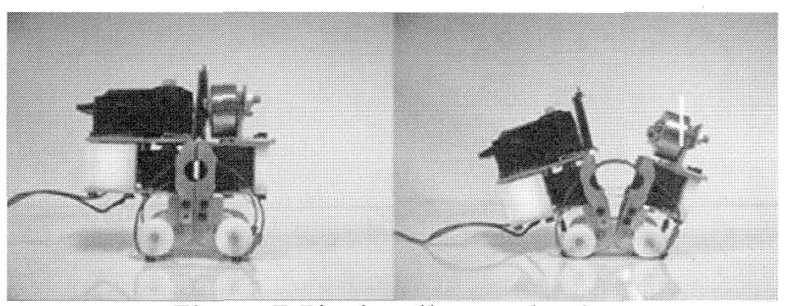

Figure 7 Pinch-roller mechanism

The key issue here is the weight of the child machine and the driving power of the cylinder used to throw the child machine. Since the child machine must be light, the piston of the cylinder starts to move with low pressure, and the flow rate cannot catch up with the internal volume expansion of the cylinder. Therefore, internal pressure fall occurs, and the driving power becomes low. As solution for this problem, a novel actuator is introduced to enhance the driving power of a pneumatic cylinder.

\section{PROPOSAL OF MAGNETIC BRAKE CYLINDER}

In order to avoid the problem of internal fall of the pneumatic cylinder and to enhance its driving power, it is necessary to increase the flow rate of air (Figure 8a, Figure $8 \mathrm{~b}$ ). The use of bigger valves, meaning using a larger effective cross-sectional area of the connection to the pneumatic cylinder, is the simplest idea, but the system becomes heavy and bulky.

To increase the flow rate of air, it is possible to use a tank connected directly to the pneumatic cylinder, and this connection can have larger effective cross-sectional area since the distance between the tank and the cylinder is small. In order to realize this method, the piston must be held in its initial position by a triggering device to avoid letting it move with low pressure (Figure 8c).

Therefore, combining the pneumatic cylinder with a tank connected directly to it, and with a triggering device, the problem of internal pressure fall can be suppressed to a minimum. As for the triggering device, a permanent magnet is used (Figure 8d). This way the trigger is passive, without the necessity of using an actuator to hold and release the piston, and it is called magnetic brake cylinder (MB Cylinder). The permanent magnet holds down the piston until the applied force to the piston by the air pressure becomes higher, and when released, it starts moving with high pressure.

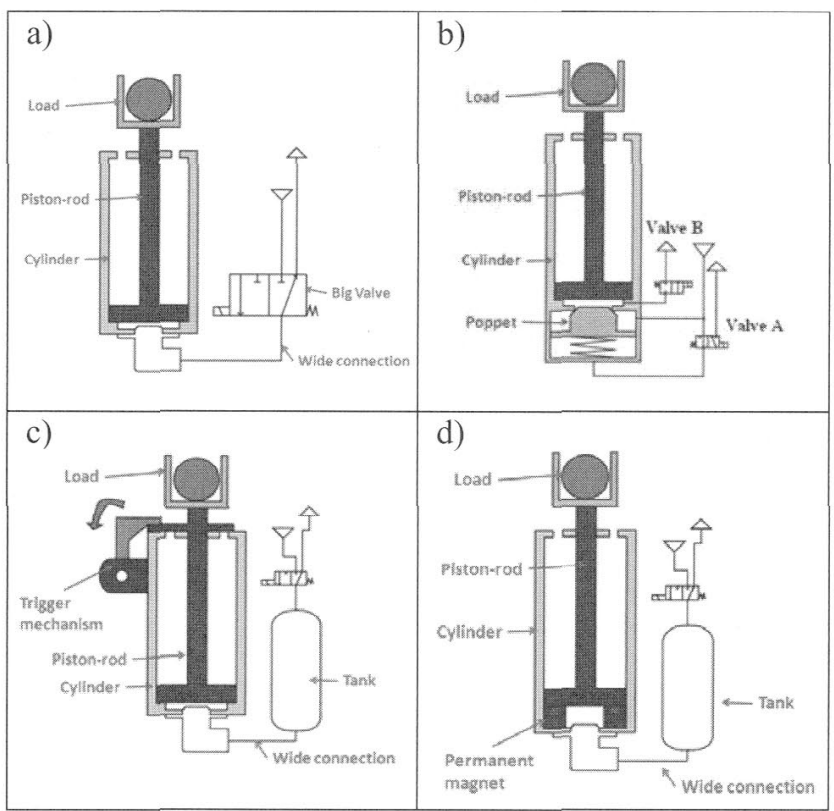

Figure 8 Possible solutions: a) bigger valve, b) poppet valve, c) triggering mechanism, d) magnetic brake cylinder

Figure 9 shows the half-cut model of the MB Cylinder. This MB Cylinder is the developed actuator for the Throw \& Collect Inspector, and it also has an additional feature to enable controlling its driving power. 


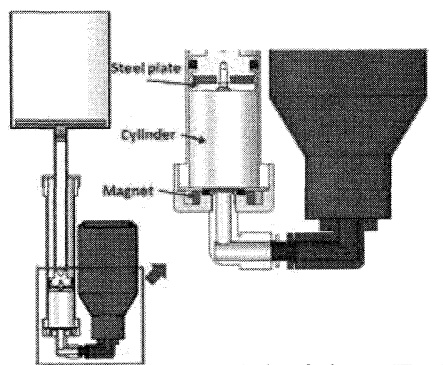

Figure 9 Half-cut model of the MB Cylinder

\section{CONTROL OF THE DRIVING POWER}

The driving power of the MB Cylinder can be controlled by two parameters: by altering the attractive force of the permanent magnet; or by altering the force applied to the piston. The first option can be realized by changing the contact area between the piston and the magnet by driving the magnet, or simply use an electromagnet to change the attractive force. However, it would be necessary big actuators, either to drive the position of the magnet or to use large coil to have significant changes to the attractive force, becoming unrealistic.

For the second option, there are two possibilities: changing the applied pressure, and change the area of the piston that receives the pressure. In order to change the applied pressure, it is necessary to use a fast pressure regulating valve, since the control must be performed after the piston detaches from the magnet, and conventional valves do not present the necessary responses for its realization. Therefore, changing the pressurized area of the piston is considered to control the driving power of the MB Cylinder. [3]

To realize this method, the inlet of the cylinder is built in such a way to create two separated chambers when the piston is in its initial position, and each chamber is pressurized by a different valve (Figure 10). The main chamber is pressurized by a main valve (valve 1 ), and only area $\mathrm{A}$ of the piston receives pressure. The control valve (valve 2) is used to pressurize area $B$ of the piston. Since area A is very small, it is necessary elevated pressure to detach the piston from the magnet, while using both areas $\mathrm{A}$ and $\mathrm{B}$, the necessary pressure becomes lower.

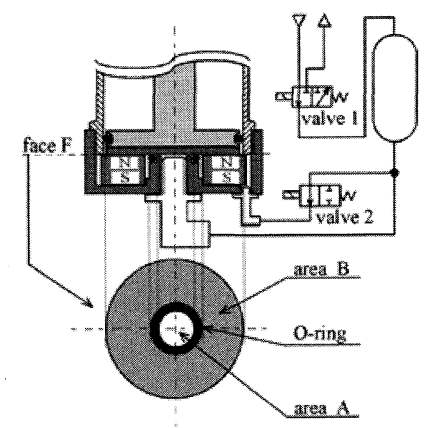

Figure 10 Inlet of cylinder divided into two chambers
Therefore, it is possible to control the driving power by timing the control valve to open in instances delayed from the main valve (Figure 11). Figure 12 shows how the internal pressure changes with different opening times, obtaining different driving powers of the $\mathrm{MB}$ Cylinder.

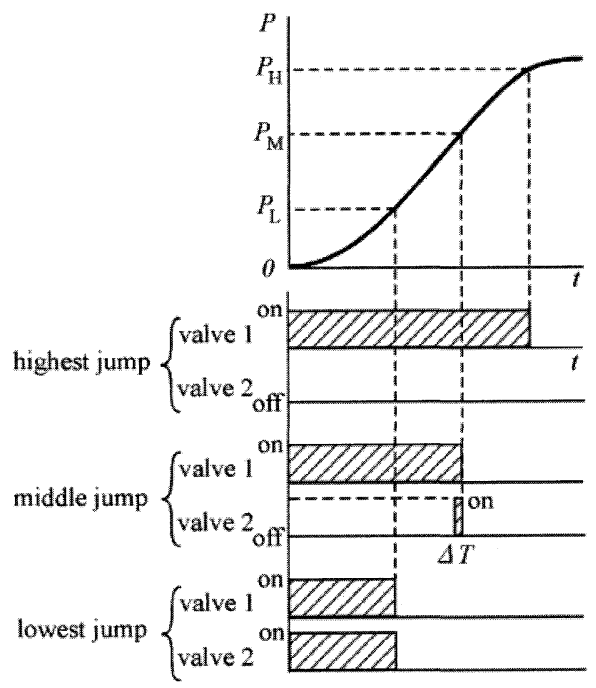

Figure 11 Time triggering of valves to control driving power

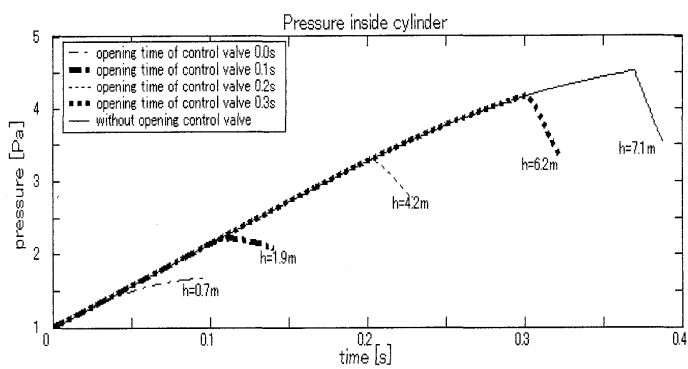

Figure 12 Internal pressure of cylinder with control method

\section{DESIGN METHOD THROUGH NUMERICAL ANALYSIS}

In reference [4], the basic equations are presented and the necessary equations to simulate the MB Cylinder regarding unknown variables are derived. Table 1 shows the parameters of the developed MB Cylinder, and in Figure 13, the simulated variables are presented with normalized values.

The simulation was validated by comparing simulated and experimental results. Figure 14 shows the results of simulation and experiment of throwing height of child machines with different masses. As observed, simulated results fit to experimental ones, and therefore, the simulation is validated. 
Table 1 System parameters

\begin{tabular}{|c|l|}
\hline \multirow{5}{*}{ Cylinder } & $A=678.87^{*} * 0^{-6}\left[\mathrm{~m}^{2}\right]$ (area of piston) \\
& $L=163 * 10^{-3}[\mathrm{~m}]$ (length of cylinder) \\
& $c=60^{*}\left(\mathrm{~A}^{*} \pi\right)^{1 / 2} \quad[\mathrm{Ns} / \mathrm{m}] \quad$ (coulomb \\
& friction coefficient) \\
& $k_{1}=220^{*}\left(\mathrm{~A}^{*} \pi\right)^{1 / 2} \quad[\mathrm{~N}] \quad$ (viscous friction \\
& coefficient) \\
& $k_{C}=100 \quad[\mathrm{~W} / \mathrm{K}] \quad$ (product of thermal \\
& conductivity and surface area of cylinder \\
& tube) \\
& $m_{r}=0.198[\mathrm{~kg}]$ (mass of rod) \\
& $m_{c}=0.360[\mathrm{~kg}]$ (mass of child machine) \\
\hline \multirow{5}{*}{ Experiment } & $g=9.81\left[\mathrm{~m} / \mathrm{s}^{2}\right]$ \\
Conditions & $P_{S}=0.46^{*} 10^{6}[\mathrm{~Pa}$ abs $]$ \\
& $P_{h}=0.1 * 10^{6}[\mathrm{~Pa}$ abs $]$ \\
& $T_{0}=293[\mathrm{~K}]$ \\
\hline \multirow{5}{*}{ Air } & $R=287[\mathrm{~J} / \mathrm{kg} \mathrm{K}]$ \\
Parameters & $c_{V}=716[\mathrm{~J} / \mathrm{kg} \mathrm{K}]$ \\
& $\kappa=1.402$ \\
& $\rho=1.293\left[\mathrm{~kg} / \mathrm{m}^{3}\right]$ \\
\hline
\end{tabular}

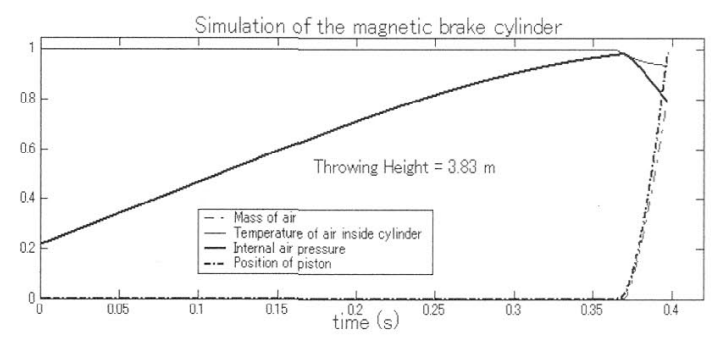

Figure 13 Simulation of MB Cylinder

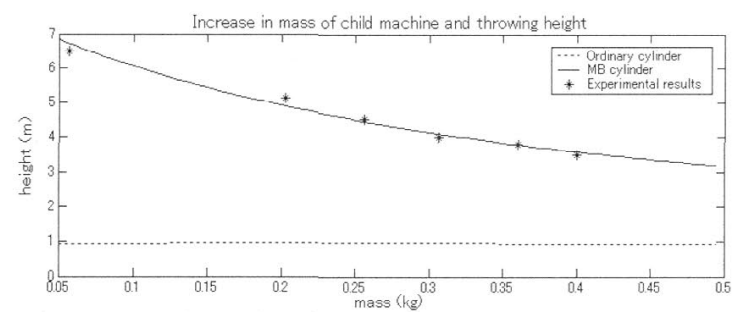

Figure 14 Throwing height of child machines with different masses

\section{Optimization Process}

The optimization of the size of the structure of the MB Cylinder is performed regarding the size of the tank and the size of the effective cross-sectional area of the connection between the tank and the cylinder.

First, the size of the tank is analyzed. Using the numerical simulation, the throwing height is observed when the volume of the tank is increased. The optimum tank volume is between 3 to 4 times the volume of the cylinder (Figure 15), and this result is equivalent to any cylinder volume. In this graph, the chosen tank volume is showed, which the volume is closest to the optimum volume, when using commercialized coffee can as the tank for the MB Cylinder.

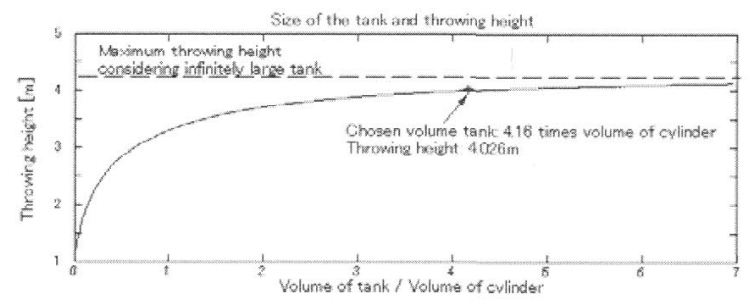

Figure 15 Increase of tank volume and throwing height

Next, the size of the connection is decided with similar process (Figure 16). The throwing height is observed when the effective cross-sectional area of the connection between the tank and the cylinder is increased. Setting $90 \%$ of the ideal throwing height, the optimum effective cross-sectional area is defined, and the connection between the tank and the cylinder is built in order to achieve this cross-sectional area.

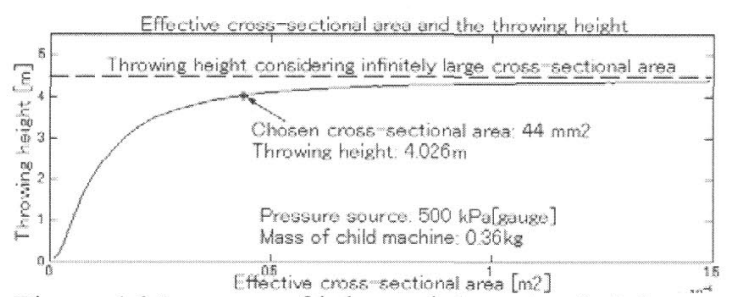

Figure 16 Increase of inlet and throwing height

With this process, the optimum size of the MB Cylinder is obtained.

\section{APPLICATION TO TARGET LOCOMOTION METHODS}

\section{Throw \& Collect Type Rescue Inspector}

With the MB Cylinder, a child machine of $0.35 \mathrm{~kg}$ is able to be thrown from a mobile inspector, which has the same structure as the Roll and Jump Mobile Inspector. Figure 17 shows the parent robot throwing the child machine over a wall of $2 \mathrm{~m}$ high, inspecting the area beyond it.

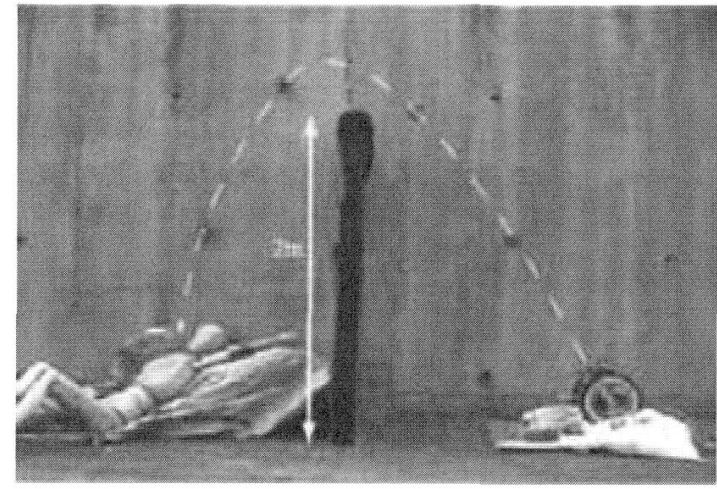

Figure 17 Throw \& Collect Mobile Inspector

The developed MB Cylinder is also mounted onto a 
pole type Throw \& Collect rescue inspector. Figure 18 shows the inspector performing a search inside a train wagon.
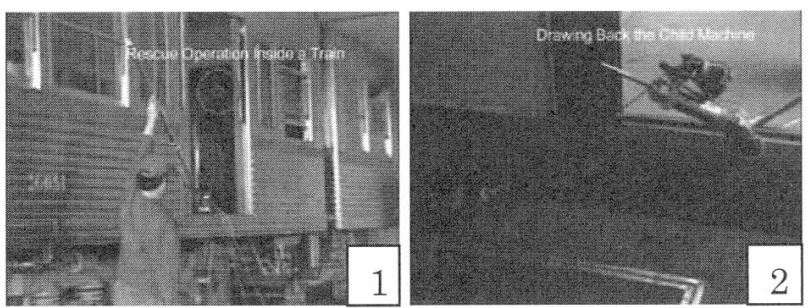

Figure 18 Inspection inside a train wagon

With the controlling method, it is also possible to access difficult places as in Figure 19. The experiment of controlling the throwing distance is showed in Figure 20, performed after using a laser range finder to measure distance and angle of the throw.

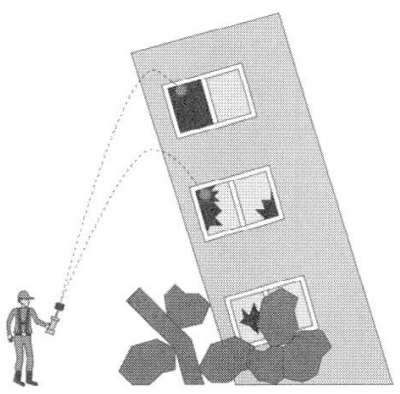

Figure 19 Enhancement of accessibility with the control method

Finally, the MB Cylinder is used to throw a child machine into a window located at the third floor of a building, as proposed in the concept (Figure 21).

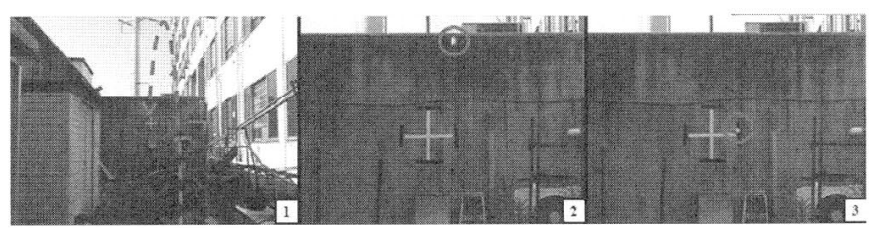

Figure 20 Throwing to the target at a distance of $18 \mathrm{~m}$

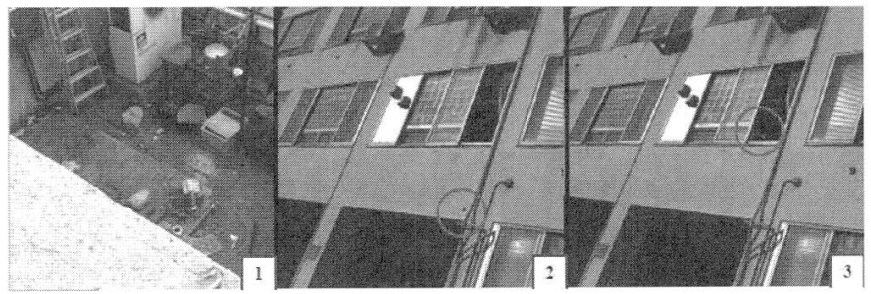

Figure 21 Throw of a child machine to inspect the third floor

\section{Roll and Jump Mobile Rescue Inspector}

The previous prototype used an ordinary pneumatic cylinder, and it was able to carry only a microcamera in order to keep the structure as light as possible. Weighting less than $2 \mathrm{~kg}$, it performed jumps of $1 \mathrm{~m}$ high.
The new prototype is smaller and heavier than the past models, being able to carry more types of sensors. It is also protected with cushion material (soft polyurethane sponge), resisting to aggressive landing. If an ordinary cylinder of same size is mounted, the robot is only able to jump $90 \mathrm{~mm}$, but changing the actuator to a MB Cylinder, the robot can jump over $1 \mathrm{~m}$ high (Figure 22).

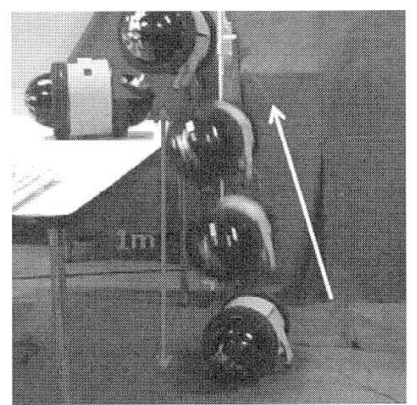

Figure 22 New prototype of the Roll and Jump Mobile Inspector

\section{SUMMARY}

A novel pneumatic actuator, the MB Cylinder, is proposed, showing to be an effective enhancement option of obstacle traversing ability. Its control method and structure is also presented. Optimization through numerical simulation is also performed.

The MB Cylinder is mounted into a Throw \& Collect rescue inspector, and the control of the throwing height proved to be useful for accessing difficult disaster areas.

The new prototype of Roll and Jump mobile inspector can perform a $1 \mathrm{~m}$ high jump being heavier than past models.

\section{REFERENCES}

1. Tsukagoshi, H., et al, "Development of Jumping \& Rolling Inspector to Improve Debris-Traverse Ability," Journal of Robotics and Mechatronics, 2003, vol.15, No.5, pp.482-490.

2. Tsukagoshi, H., et al, "Development of a Throwing \& Drawing Type Inspector Aimed to Wide-Range Searching Inside Collapsed Buildings," 2005 JSME Conference on Robotics and Mechatronics, 2005, 1P2-S-096.

3. Watari, E., et al, "Development of a Throw \& Collect Type Rescue Inspector $-6^{\text {th }}$ Report: Control of the Throwing Distance by a Magnetic Brake Cylinder," SICE-ICASE International Joint Conference 2006, SICE-ICCAS 2006, 2006, SA08-4.

4. Tsukagoshi, H., et al, "Design of a Higher Jumping Rescue Robot With the Optimized Pneumatic Drive," Proceedings of the 2005 IEEE International Conference on Robotics and Automation, 2005, pp. 1288-1295. 